\section{GLINT: AUdIOVISUAL GLITCHES}

Funda Senova Tunali, Bilkent University, Institute of Fine Arts, Department of Graphic Design, Bilkent, 06800, Ankara, Turkey

E-mail:<sfunda@bilkent.edu.tr>.

See $<$ www.mitpressjournals.org/toc/leon/45/3> for supplemental files associated with this issue.

Submitted: 14 July 2010

\section{Abstract}

This paper explores the idea of glitch through an audiovisual project called Glint and how the concept of glitch can be marked as a manifestation of digital culture.

Glint is a 3-minute video project that combines digital typography and sound design via the concept of "glitch," conducted as a part of an ongoing PhD study [1]. The study researches occurring patterns between two domains that would imply an existing ideology of digital culture. Glitch as a malfunction and a drawback occurring in the system manifests the existence of technology, media used and the implemented interface. It occurs as a common ground and an intermediary in both visual and aural domains.

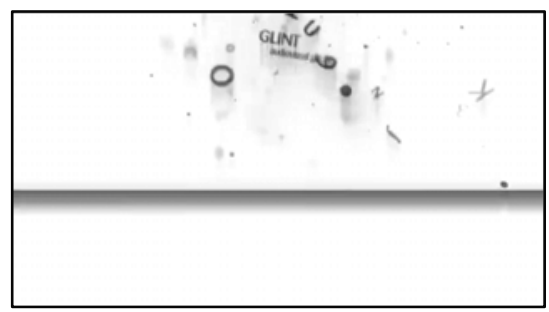

Fig. 1. Screenshot from the title sequence of Glint video. (ㄷ) Funda Senova Tunali.)

The essential motive for choosing a theme that resonates with the idea of error and malfunction is to trace a possible pattern occurring in both domains. The project was conducted in a digital environment by using image editing software, motion graphics and compositing software, music production software, audio editor and various plug-ins.

Naming the project Glint has several purposes. The name Glint carries multiple connotations with it, glint's meaning being "a glimpse, glance or gleam," "a tiny quick flash of light" and "a brief or slight manifestation or occurrence" indicate more than just a phonetical resemblance with the word glitch [2]. A glitch occurs as a momentary manifestation within the system and disappears afterwards; it is unexpected, unpredictable and has a shocking effect. The video

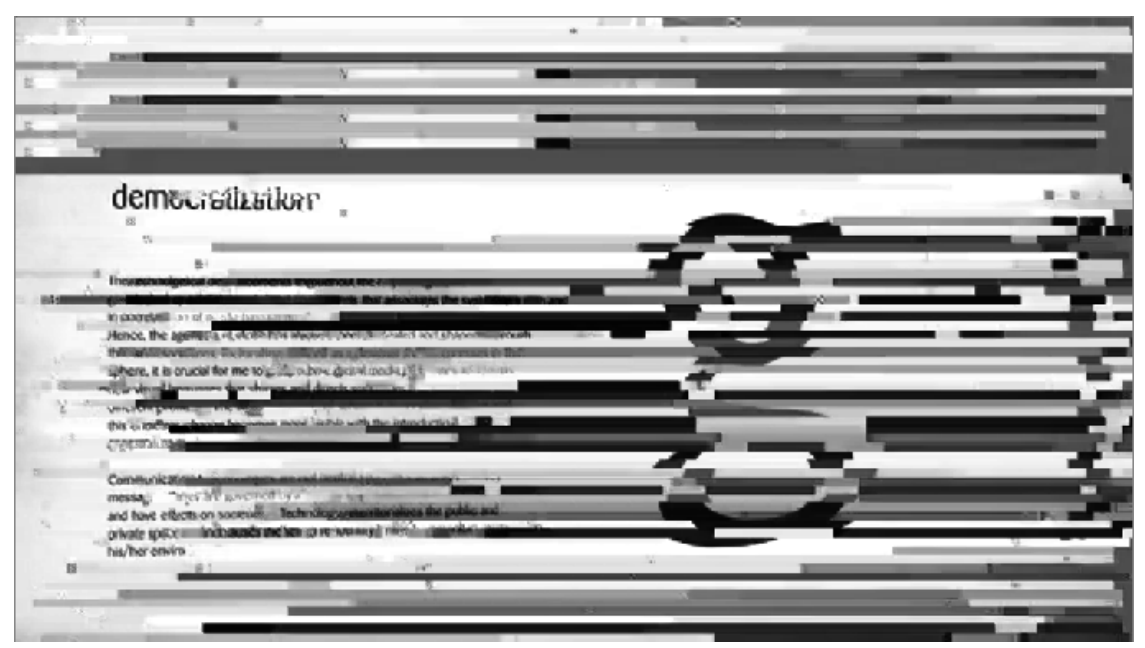

Fig. 2. Screenshot from the Glint video. (C Funda Senova Tunali.)

Glint suggests such an experience- - a narrative that is shaped by assigning glitch-alikes, which give significance and contribute to the context of the composed audio and visual materials. Glitch as a manifestation does not effect the content and its organization; however, it affects the viewing experience intensely. A glitch creates momentary alienation while creating an uncanny effect and conveying information about the media that it is manifested on. A glitch that occurs in a system diverts the attention of the user/viewer/participant, troubles $\mathrm{him} /$ her and results in a discomforting experience. Glint's aim is to produce an audiovisual experience that is stimulating and intriguing via the use of constructed glitch-alikes, which would not cause such a discomforting experience.

The number of artists exercising the concept of glitch in visual and aural domains is increasing. Some artists work with pure glitches by forcing the tools to generate them, while others work with glitch-alikes mainly by synthesizing them. Iman Moradi stated that "[i]n forcing a visual glitch, there is an element of unpredictability that makes experimentation worthwhile and rewarding" [3]. In such manner, it is worth noting that there are various ways of achieving glitches and glitch-alikes; furthermore, both pure glitches and glitch-alikes demand excessive experimentation. The designers who are working with the concept of glitch should be very well aware of the processing tools, such as computer hardware and software, and should have a clear grasp of the significance and operation of these structures not just as tools but also as materials that enhance the interactivity of the user/viewer/ participant.

The Glint project was composed by using glitch-alikes. Although glitches are undesired occurrences, the video project tries to challenge this understanding by adding the concept of intentionality, thus transforming the notion of glitch into a design element. In a designed piece, every element consistently carries intentionality. Since design is a problemsolving activity, accident or coincidence within a work are not acceptable in terms of design decisions. Design is a conscious activity, since the whole process is shaped by those decisions made by the designer. Moreover, if the objective within a work is to achieve an expression that looks accidental, then it is solely based on the pure consciousness of the designer. Hence, considering Glint, using glitches as design elements within the project brings a variant viewing experience that forms the audiovisual continuity and consistency throughout the video. Selecting a common theme such as glitch that can be applied and has already manifested itself in both domains serves as a relating aspect that forms a common audiovisual language, which is based on same principles. Thus, Glint is also based on the intentionality of the designer, although it tries to create a sense of ghost in the machine that has its own logic, operating system and out-ofcontrol feeling.

When visual glitches are generated by designers, they are considered glitchalikes because of their mimicry of pure glitches and their production methods [4]. They are the calculated, designed, formed, synthesized, faked and interpreted forms of malfunctions that are generated by the system accidentally. So Glint becomes absolutely not a project that is formed by the compilation of pure glitches. It imitates the visual and the auditory characteristics of glitches on a 
conscious level to present an alternative viewing and listening experience.

Glint tries to address the representational status quo of technology, digital media and the culture's reception and reflection of it. It attempts to represent the signifiers of digital media by implementing hypermediacy, by which the user/viewer/participant is always reminded of the existence of the medium that the content is offered through. Instead of creating an immersive and immediate experience, it is constructed upon the principle of hypermediacy, where the user is always made aware of the technology - in a sense made aware of the window/display with which s/he uses to reach out to the content by remediating the data as a conscious decision made, using a fault, a drawback of that very same digital technology. The glitches act as actualizations of many possible manifestations, which the user of digital technologies can encounter any time.

The analysis of Glint is aimed at revealing similar patterns of production, development, distribution, and consumption. When the project is broken up into pieces and analyzed, each part can be considered as a system with which assumptions can be made about a larger system that encompasses the relatively small ones.

The analysis of the project consists of the study of the preparation, growth of research, accessing and processing knowledge, generation of ideas, making of sketches and storyboards, transformation of such ideas into visual and audio materials, the software and hardware used in this process, the association of these software, the GUI that the designer and the viewer are confronted with throughout the process, compilation of the audio and video tracks, distribution

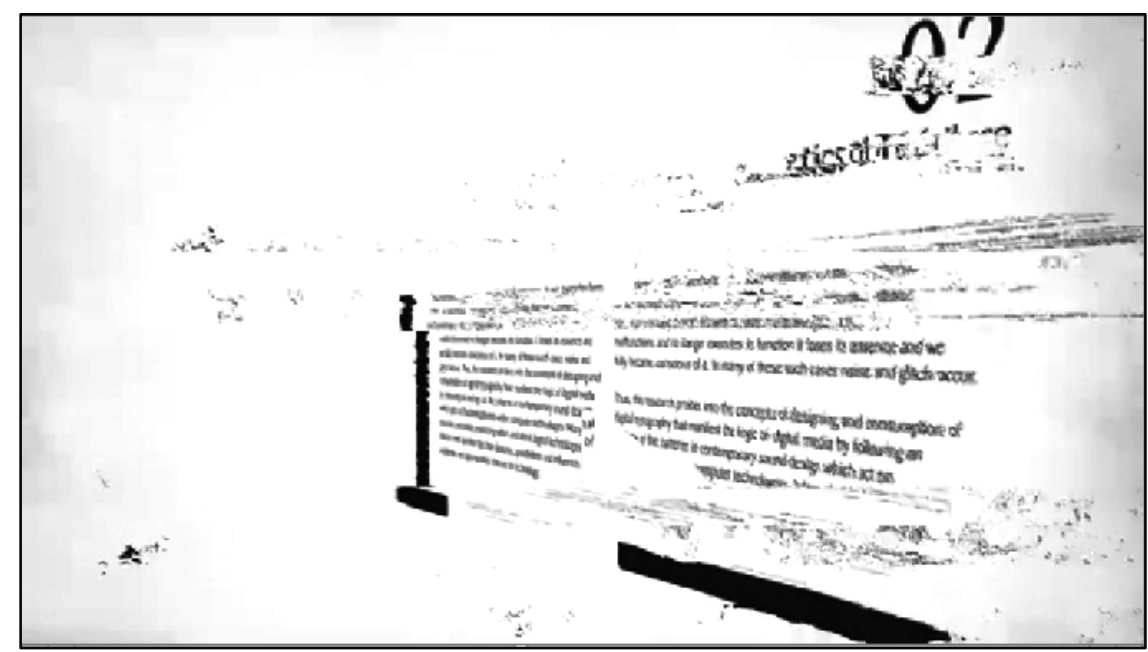

Fig. 3. Screenshot from the Glint video. (C Funda Senova Tunali.)

channels and means, the distributed signifiers, the relationship between these signifiers and the status quo, people who consume this project by accessing, watching and reflecting on it, and the culture that is originated by these consumers. The analysis of each of these stages and elements is aimed at manifesting the digital culture, which has its own rules and ideology - thus, has an operational logic.

Digital culture is shaped by the role of the designer; the assumed responsibility that $\mathrm{s} /$ he undertakes, the equipment used in achieving this goal, the distribution channels and the ways in which the production is utilized and consumed are different indicators of digital culture. So the process from the production to the consumption of the project indicates what Manovich calls a cultural interface, where people can interact with data through the use of computers [5]. Although the video itself is a complete, closed form where no additional data can be used once it is finished and rendered, the way that is produced, distributed and accessed are designations of open forms and they welcome feedback mechanisms. Thus, it also has implications of interactivity and participation. To this extent, Glint manifests itself not just as an audiovisual project but also as a project pinpointing the continuum from production to consumption of digital outputs.

\section{References and Notes}

1. An extended version of this paper appears as a sub-chapter of a PhD dissertation that will be submitted to Bilkent University, Institute of Fine Arts, Department of Graphic Design.

2. Dictionary.com <www.dictionary.com>, accessed July 2010.

3. I. Moradi, A. Scott, J. Gilmore \& C. Murphy Glitch: Designing Imperfection (New York: Mark Batty Publisher, 2009) p. 9.

4. I. Moradi, Glitch Aesthetics (UK: University of Huddersfield, 2004) p. 8.

5. C-O. Wong, K. Jung and J. Yoon, "Interactive Art: The Art that Communicates," Leonardo 42, No: 2, 180-181 (2009).

6. L. Manovich, The Language of New Media (Cambridge, Massachusetts, 2001). 


\section{Thanks to Our Supporters}

Leonardo/ISAST is a nonprofit organization that serves the international arts community by documenting work at the intersection of the arts, sciences and technology and by encouraging and stimulating collaboration through its programs and activities. Donations and grants are integral to the future of Leonardo.

Contact<isast@leonardo.info> or visit <http://leonardo.info> for more information.

Leonardo Codex

$(\$ 5,000$ and above $)$

College of Extended Learning,

San Francisco State University

Roger Malina

The Malina Trust

Sonya Rapoport

Rockefeller Foundation

The San Francisco Art Institute

Al Smith

Darlene Tong

University of Texas at Dallas

\section{Sforza Monument}

(The Bronze Horse)

$(\$ 1,000$ to $\$ 4,999)$

Martin Anderson

Art Science Research Lab

Banff New Media Institute

Lisa Bornstein

CalArts

Caldas University Arts

and Humanities

Concordia University

DeMontfort University Institute for

Creative Technologies

Emily Carr University of Art \& Design

Donna Cox

Creative Disturbance

The Daniel Langlois Foundation

Char Davies

The Exploratorium

Penny Finnie

Steve Forestieri

Gregory Harper

John Hearst

Intel Corporation

LABoral Centro de Arte y Creacion Industrial

The LEF Foundation

Alan Malina

Marjorie Malina

Jacques Mandelbrojt

Christine Maxwell-Malina

Ontario College of Art \& Design University

Sheila Pinkel

Michael Punt

Rhode Island School of Design

Itsuo Sakane

School of the Art Institute of Chicago

Scottsdale Center for the Performing Arts

Martin Segal

SFSU International Center for the Arts

Sonia Sheridan

Srishti School of Art, Design and Technology

swissnex San Francisco

SymbioticA

Marcia Tanne

Makepeace Tsao

UCLA Art / Sci Center

UC Santa Barbara, Media Arts and Technology Program

UC Santa Cruz, Digital Arts and

New Media Dept.

Universidad Autonoma de Occidente,

Engineering Department

Universidad de San Buenaventura,

Multimedia Engineering Program

Universidade de Caldas

Universidade do Minho

University of Calabria Evolutionary Systems Group

University of Denver School of Art

and Art History
University of Évora, CHAIA

University of Florida Digital Worlds Institute

University of Illinois

eDREAM Institute

University of Leiden

University of Plymouth, U.K.

University of Washington DXARTS

UTS Creativity and Cognition Studios

ZKM | Center for Art and Media

\section{La Gioconda (Mona Lisa)}

(\$500 to \$999)

Roy Ascott

Lars Ole Belhage

Martha Blassnigg

Anna Campbell Bliss

Leif Brush

James D. Burke

Richard Clar

Una Dora Copley

Bryony Dalefield

Michele Emmer

William Fawley

Arana Greenberg

Michael Joaquin Grey

Dene Grigar

Rosemary Jackson

Larry Larson

Lynn Hershman Leeson

Guy Levrier

Isabel Maxwell

Merrill Lynch Foundation

Emanuel Nadler

Nessim \& Associates

Sam Okoshken

Steve Oscherwitz

Trudy Reagan

David Rosenboom

Jack Sarfatti

Joel Silverman

Christian Simm

Tami Spector

Meredith Tromble

\section{Flying Machine}

\section{(\$250 to \$499)}

Loren Basch

Ray Bradbury

Bettina Brendel

Shawn Brixey

David Carrie

Eva Craig

Holly Crawford

Eugene Epstein

Lawrence Fane

Herbert Franke

Doreen Gatland

Pamela Grant-Ryan

Oliver Grau

Linda Dalrymple Henderson

Robert Hill

Curtis Karnow

Melinda Klayman

Kathleen Laziza

Thomas Mercer

Gianluca Mura

Frieder Nake

Barbara Nessim

Jack Ox

Ed Payne and Liss Fain

Nancy Perloff

Frank Popper

Harry Rand

\author{
Beverly Reiser \\ Mark Resch \\ Eric Roll \\ Edward Shanken \\ Leonard Shlain \\ Jesse Tischler \\ Joan Truckenbrod \\ Kelvin Tsao \\ Jonathan Willard \\ Barbara Lee Williams \\ Richard A. Wilson \\ Stephen Wilson
}

Gary Zellerbach

\section{Angel}

(\$249 and under)

Anonymous, Aaron Alpar, Charles Ames, Craig Anderson, Art Science Collaborations Inc. (ASCI), Yasuhiro Asoo, Bret Battey, Marc Battier, Mark and Lauren Beam, Patricia Bentson, Timothy Binkley, The Birse Family, Marc Böhlen, Deborah Branton, Robert A. Brown, Ronald Brown, Willi Bruns, Annick Bureaud, James Burke, David Carter, Rosa Casarez-Levison, Webster Cash, Katherine Casida, Joel Chadabe, Alison Chaiken, John Chowning, Richard Clar, Computer Art

Studio/Gunter Schulz, Ivo Cristante, Elizabeth Crumley, Mary \& Michael Cunningam, Danish Film Festival, Bob Davis, Derrick de Kerckhove, Goery Delacote, Lily Diaz, Agnes Denes, Emma Lou Diemer, Steve Dietz, Augus Dorbie, Hubert Duprat, Elmer Duncan, Ann Elias, Sherban Epure, Theodosia Ferguson, John Fobes, Tim Fox, Alan \& Mickey Friedman, Ryozo Fujii, Kai-hung Fung, David Gamper, Jonathan \& Donna R. Gennick, George Gessert, Ken Goldberg, Yusef Grillo, Karen Guzak, Craig Harris, Isabel Hayden, Margaret Hermann, Doris Herrick, Estate of Dick Higgins, Kathy High, Anthony Hill, Toshivuki Hiruma, Gerald Holton, Hungarian University of Crafts \& Design, Amy Ione, Susan Joyce, Raymond Jurgens, Eduardo Kac, Robert Kadesch, Marshall Kaplan, Ken Knowlton, Zdenek Kocib, Kenji Kohiyama, Thomas Kostusiak, Kathleen Laziza, Levi Family Foundation, Kathleen La:iza, Le Carl Machover, James Maher, William Marchant, Delle Maxwell, Maher, William Marchant, Delle Maxwell,
Elliot Mazer, Kevin Meehan, Minneapolis College of Art \& Design, Mit Mitropoulos, Moët Hennessy-Louis Vuitton, Jason Monberg, Roger Mulkey, Geetha Narayanan, Alex Nicoloff, Greg Niemeyer, Hiroshi Ninomiya, Elaine Petschek, Anne Brooks Pfister, Glenn R. Phillips, Victor A. Pickett, Otto Piene, Ann Pizzorusso, Herbert \& Joan Webster Price, Patric Prince, Wolf Rainer, Peter Richards, Ron Rocco, Peter Rudolfi, David M. Russell, Mr. and Mrs. Robert Russett, Colin Sanderson, Piero Scaruff, Patricia Search, Allan Shields, Gregory C. Shubin, Joel Slayton, John Slorp, Avril Sokolov, Kirill Sokolov, Christa Sommerer, Rejane Spitz, Anait Stephens, Robert Strizich, The Sun Microsystems Foundation, Inc., Robin and Barbara Tchartoff, Tamiko Thiel, Rodrigo B. Toledo, Heinz Trauboth, Mark Tribe, Karen Tsao, Roman Verostko, Alexandre Vitkine, Annette Weitraub, Natalie \& Mark Whitson, Alan Thompson \& Sharon A. Widmayer, Ioannis Yessios, Robert Zimmerman 